CRYSTALLOGRAPHIC COMMUNICATIONS

ISSN 2056-9890

Received 23 June 2020

Accepted 9 July 2020

Edited by J. T. Mague, Tulane University, USA

Keywords: crystal structure; silver sulfonate salt; low temperature; hydrogen bonding.

CCDC reference: 2015332

Supporting information: this article has supporting information at journals.iucr.org/e

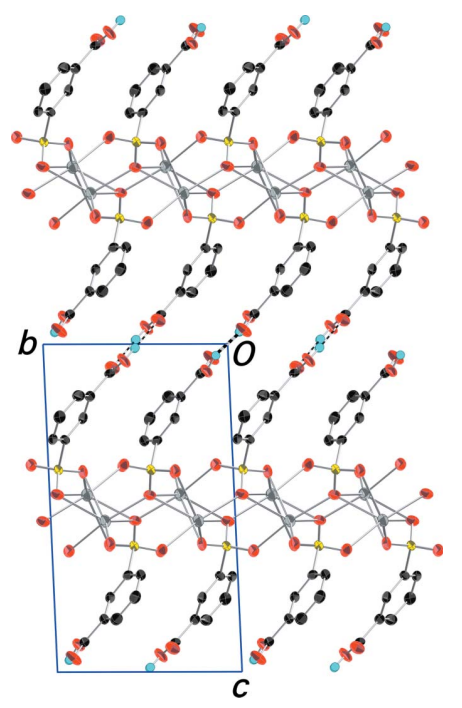

OPEN $\odot$ ACCESS

\section{The low-temperature triclinic crystal structure of silver 3-sulfobenzoic acid}

\author{
Reuben T. Bettinger, ${ }^{a}$ Philip J. Squattrito ${ }^{a}{ }^{a}$ and Darpandeep Aulakh ${ }^{b}$
}

${ }^{a}$ Department of Chemistry and Biochemistry, Central Michigan University, Mount Pleasant, Michigan 48859, USA, and ${ }^{\mathbf{b} C o l l e g e}$ of Natural Sciences and Mathematics, University of Toledo, Toledo, OH 43606, USA. *Correspondence e-mail: p.squattrito@cmich.edu

Poly[ $\left(\mu_{4}-3\right.$-carboxybenzenesulfonato $)$ silver(I) $], \mathrm{Ag}\left(\mathrm{O}_{3} \mathrm{SC}_{6} \mathrm{H}_{4} \mathrm{CO}_{2} \mathrm{H}\right)$ or $\left[\mathrm{Ag}\left(\mathrm{C}_{7} \mathrm{H}_{5^{-}}\right.\right.$ $\left.\left.\mathrm{O}_{5} \mathrm{~S}\right)\right]_{n}$, has been found to undergo a reversible phase transition from monoclinic to triclinic between 160 and $150 \mathrm{~K}$. The low-temperature triclinic structure (space group $P \overline{1}$ ) has been determined at $100 \mathrm{~K}$. In contrast to the reported room temperature monoclinic structure, in which the nearly equivalent carboxylate $\mathrm{C}-\mathrm{O}$ distances indicate that the acidic hydrogen is randomly distributed between the $\mathrm{O}$ atoms, at $100 \mathrm{~K}$ the $\mathrm{C}-\mathrm{O}$ (protonated) and $\mathrm{C}=\mathrm{O}$ (unprotonated) bonds are clearly resolved, resulting in the reduction in symmetry from $C 2 / c$ to $P \overline{1}$.

\section{Chemical context}

Over the past two decades, organosulfonate and organocarboxylate anions have received significant attention as building blocks for metal-organic framework (MOF) structures (Dey et al., 2014; Shimizu et al., 2009). As a result of its soft nature, sulfonate tends to bond well with soft cations like silver(I) so a significant chemistry of silver sulfonates has developed during this period (Côté \& Shimizu, 2004; Hoffart et al., 2005). Having previously investigated some structures of silver sulfonate salts (Downer et al., 2006; Squattrito et al., 2019), we have continued this effort with the reaction of $\mathrm{Ag}^{+}$ with the bifunctional 3-sulfobenzoate anion. The resulting monobasic salt has been found to have an unexpected lowtemperature structural modification that is reported here.<smiles>O=C(O)c1cccc(S(=O)(=O)[OH2+])c1</smiles>

\section{Structural commentary}

The product of the reaction of silver nitrate and sodium 3-sulfobenzoic acid is $\mathrm{Ag}\left(\mathrm{O}_{3} \mathrm{SC}_{6} \mathrm{H}_{4} \mathrm{CO}_{2} \mathrm{H}\right)$, (I), an anhydrous monobasic silver(I) salt of 3-sulfobenzoic acid. The roomtemperature $(293 \mathrm{~K})$ structure of (I) was previously reported in the monoclinic space group $C 2 / c$ with one independent cation and anion in the asymmetric unit (Prochniak et al., $2008)$. We find the structure at $100 \mathrm{~K}$ to be triclinic $(P \overline{1})$ with two independent cations and anions in the asymmetric unit (Fig. 1). The major features of the structure at $100 \mathrm{~K}$ are consistent with those at $293 \mathrm{~K}$. The silver ions are coordinated 
Table 1

Selected bond lengths $(\AA)$.

\begin{tabular}{llll}
\hline $\mathrm{Ag} 1-\mathrm{O} 3^{\mathrm{i}}$ & $2.3868(18)$ & $\mathrm{Ag} 2-\mathrm{O} 9^{\mathrm{ii}}$ & $2.4090(17)$ \\
$\mathrm{Ag} 1-\mathrm{O} 8$ & $2.4091(18)$ & $\mathrm{Ag} 2-\mathrm{O} 5^{\mathrm{iv}}$ & $2.4199(18)$ \\
$\mathrm{Ag} 1-\mathrm{O} 10^{\mathrm{ii}}$ & $2.4406(18)$ & $\mathrm{Ag} 2-\mathrm{O} 4^{\mathrm{v}}$ & $2.4609(18)$ \\
$\mathrm{Ag} 1-\mathrm{O} 10^{\mathrm{iii}}$ & $2.5249(18)$ & $\mathrm{Ag} 2-\mathrm{O} 4$ & $2.5295(18)$ \\
$\mathrm{Ag} 1-\mathrm{O} 5$ & $2.6853(19)$ & $\mathrm{Ag} 2-\mathrm{O} 8$ & $2.6953(19)$ \\
$\mathrm{Ag} 1-\mathrm{O} 4$ & $2.7254(19)$ & $\mathrm{Ag} 2-\mathrm{O} 10$ & $2.7179(19)$ \\
\hline
\end{tabular}

Symmetry codes: (i) $-x+3,-y+1,-z+1$; (ii) $-x+2,-y,-z+1$; (iii) $x+1, y, z$; (iv) $x-1, y, z$; (v) $-x+2,-y+1,-z+1$.

by six sulfonate $\mathrm{O}$ atoms with four shorter ( ca 2.4-2.5 $\AA$ ) and two longer ( $c a$ 2.7 $\AA$ ) distances (Table 1) in an irregular hexacoordinate geometry [somewhat inaccurately described as tetrahedral by Prochniak et al:; the $\mathrm{O}-\mathrm{Ag}-\mathrm{O}$ angles for the four shorter $\mathrm{Ag}-\mathrm{O}$ bonds range from 71.25 (7) to $164.88(6)^{\circ}$ indicating at best a very distorted tetrahedron]. Not surprisingly, the $\mathrm{Ag}-\mathrm{O}$ distances are shorter by an average of $0.02 \AA$ at $100 \mathrm{~K}$ than at $293 \mathrm{~K}$. This kind of pseudotetrahedral coordination geometry significantly distorted by two somewhat longer $\mathrm{Ag}-\mathrm{O}$ interactions was previously observed in the silver salt of 6-ammonionaphthalene-1,3-disulfonate (Downer et al., 2006). The $\mathrm{Ag}-\mathrm{O}$ distances are consistent with those seen in other silver arenesulfonates (Côté \& Shimizu, 2004). The extensive metal-sulfonate bonding is as expected given the softer nature of $\mathrm{Ag}^{+}$relative to most $d$-block transition-metal ions (Parr \& Pearson, 1983), which generally show little tendency to bond directly to sulfonate groups (Ma et al., 2003). The carboxylate group remains protonated with the acidic $\mathrm{H}$ atoms unambiguously located on $\mathrm{O} 2$ and $\mathrm{O} 7$. The $\mathrm{C}-\mathrm{O}$ distances in the carboxylate groups clearly distinguish the non-protonated $(\mathrm{C}=\mathrm{O})$ and protonated $(\mathrm{C}-\mathrm{O}) \mathrm{O}$ atoms: C7-O1 1.232 (3), C7-O2 1.312 (3) Å; C14-O6 1.231 (3), C14-O7 1.311 (3) A.

\section{Supramolecular features}

The packing in (I) features layers of metal ions in the $a b$ plane alternating with double-layers of 3-sulfobenzoic acid anions

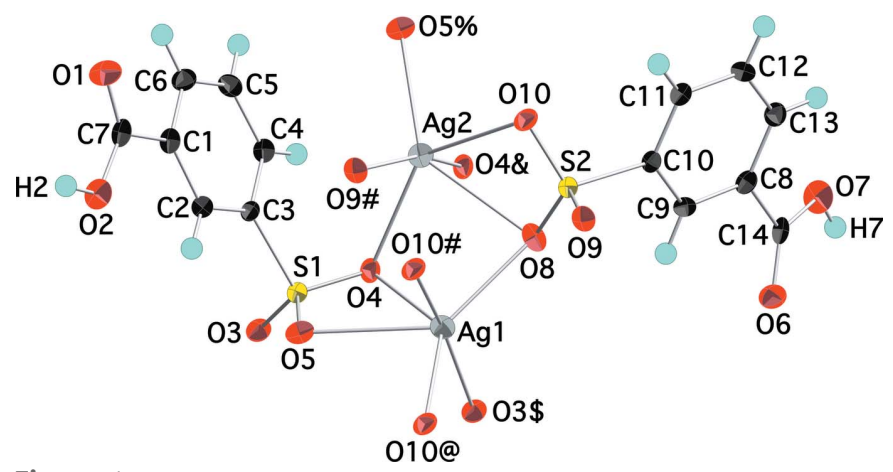

Figure 1

The molecular structure of (I), showing the atom-numbering scheme. Displacement ellipsoids are shown at the $75 \%$ probability level and hydrogen atoms are shown as small spheres of arbitrary radii. Symmetryequivalent oxygen atoms are included to show the complete coordination environments of the cations. [Symmetry codes: (\#) $2-x,-y, 1-z$; (@) $x+1, y, z ;(\$) 3-x, 1-y, 1-z ;(\&) 2-x, 1-y, 1-z ;(\%) x-1, y, z$.]
Table 2

Hydrogen-bond geometry $\left(\AA,{ }^{\circ}\right)$.

\begin{tabular}{|c|c|c|c|c|}
\hline$D-\mathrm{H} \cdots A$ & $D-\mathrm{H}$ & $\mathrm{H} \cdots A$ & $D \cdots A$ & $D-\mathrm{H} \cdots A$ \\
\hline $\mathrm{O} 2-\mathrm{H} 2 \cdots \mathrm{O} 1^{\mathrm{vi}}$ & 0.84 & 1.81 & $2.631(3)$ & 164 \\
\hline $\mathrm{O} 7-\mathrm{H} 7 \cdots \mathrm{O}^{\mathrm{vii}}$ & 0.84 & 1.81 & $2.651(3)$ & 176 \\
\hline
\end{tabular}

Symmetry codes: (vi) $-x+2,-y,-z$; (vii) $-x+2,-y+1,-z+2$.

stacking along the $c$-axis direction (Fig. 2). Anions in adjacent layers are linked by $\mathrm{O}-\mathrm{H} \cdots \mathrm{O}$ hydrogen bonds between neighboring carboxylic acid groups in the classic dimerization of such molecules (Table 2; Fig. 3). The symmetry-independent anions alternate in the $b$-axis direction within the layer. The rings of these anions are significantly out of parallel with an interplanar angle of $c a 139^{\circ}$. This packing motif with the

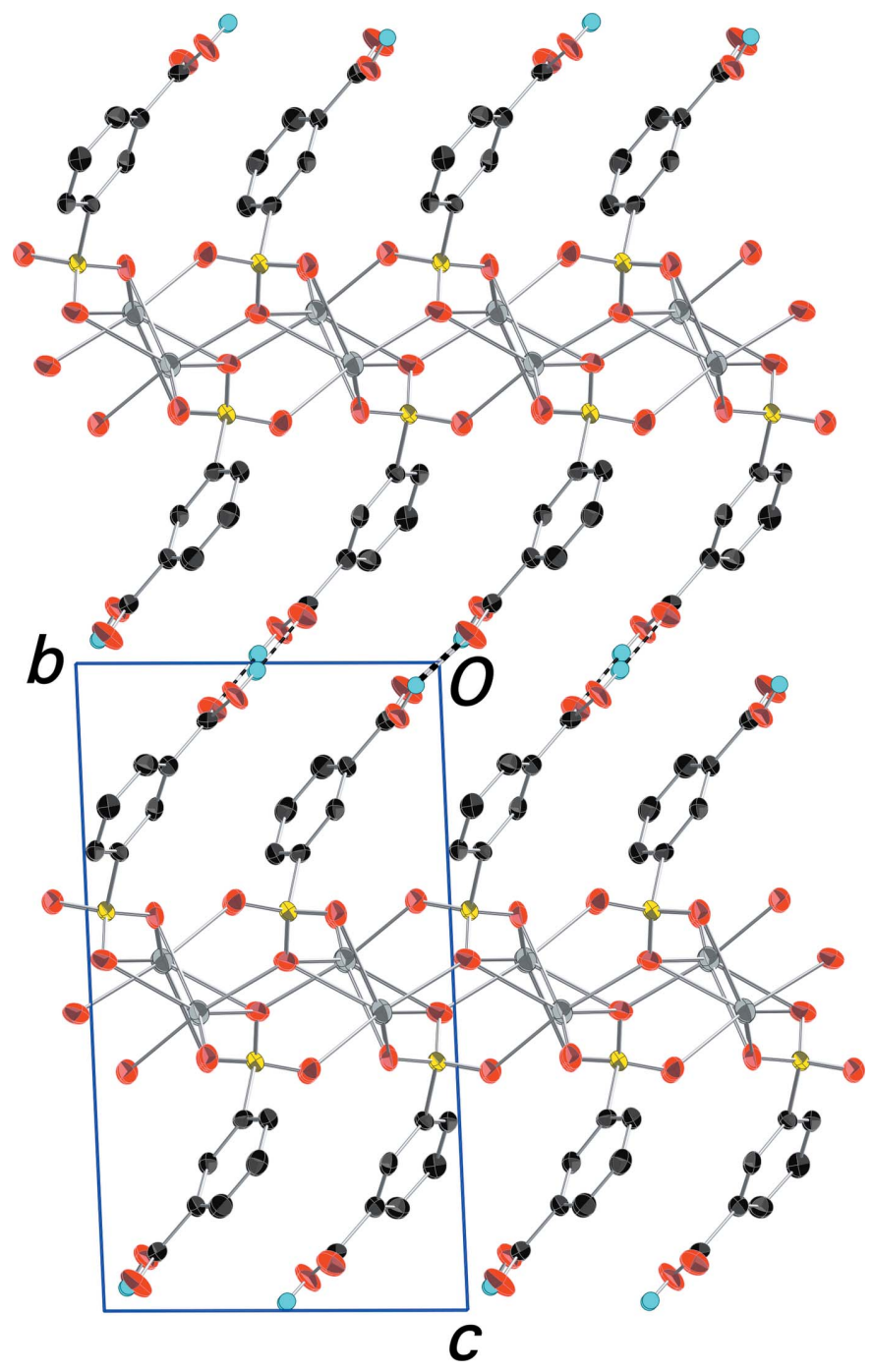

Figure 2

Packing diagram of (I) with an outline of the unit cell. View is onto the (100) plane. The double-layers of 3-sulfobenzoic acid anions are evident with the silver ions situated in between the layers. $\mathrm{O}-\mathrm{H} \cdots \mathrm{O}$ hydrogen bonds connecting the carboxylic $\mathrm{H}$ atoms and carboxylate $\mathrm{O}$ atoms of adjacent layers are shown as dashed bonds. $\mathrm{H}$ atoms bonded to $\mathrm{C}$ atoms have been omitted. Displacement ellipsoids are drawn at the $90 \%$ probability level. 


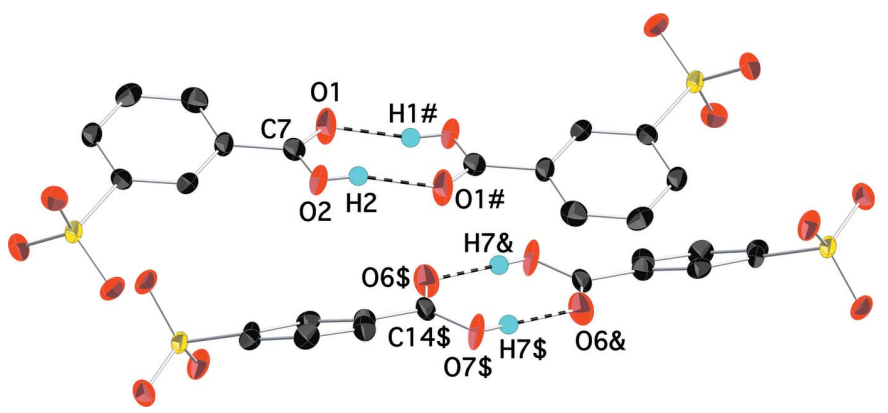

Figure 3

Partial packing diagram of (I) showing the hydrogen-bonding scheme involving the carboxylic acid groups of neighboring anions. Hydrogen bonds are shown as dashed bonds. Displacement ellipsoids are drawn at the $90 \%$ probability level. [Symmetry codes: (\#) $2-x, 1-y, 1-z$; (\$) $3-x, 2-y, 2-z ;(\&) x+1, y+1, z]$

sulfonate and carboxylate groups directed to opposite sides of the layer is contrary to what was found in the silver salt of the isomeric 4-sulfobenzoic acid (Squattrito et al., 2019). In that compound, both functional groups are involved in metaloxygen bonding so the anions are positioned with both groups equally distributed with respect to each surface of the layer, in contrast to the segregated arrangement in (I).

Comparison of the $100 \mathrm{~K}$ and $293 \mathrm{~K}$ structures reveals that the key difference is in the carboxylate group. At $293 \mathrm{~K}$, the $\mathrm{C}-\mathrm{O}$ bond lengths are almost the same [1.250(3) and $1.271(3) \AA]$, indicating significant disorder between the protonated and non-protonated $\mathrm{O}$ atoms, while at $100 \mathrm{~K}$ the $\mathrm{C}-\mathrm{O}$ and $\mathrm{C}=\mathrm{O}$ bonds are clearly distinguished and the placement of the acidic $\mathrm{H}$ atoms accordingly renders the two 3 -sulfobenzoic acid moieties symmetry-inequivalent. Variabletemperature single-crystal X-ray measurements between 250 and $130 \mathrm{~K}$ show that the monoclinic-to-triclinic transition occurs on going from 160 to $150 \mathrm{~K}$ and that it is reversible.

\section{Database survey}

A search of the Cambridge Structural Database (CSD, Version 5.41, update of November 2019; Groom et al., 2016) for metal 3-sulfobenzoate salts that do not contain aromatic rings containing nitrogen (aromatic amines are popular secondary linkers in MOF systems) yielded twenty hits. Of these, eleven contain other amines. The nine reported structures containing only metal ions and 3-sulfobenzoate ions (protonated or unprotonated), with or without water molecules, are the $293 \mathrm{~K}$ structure of (I) (refcode ROJJUW; Prochniak et al., 2008), sodium 3-sulfobenzoic acid dihydrate (ROJJOQ; Prochniak et al., 2008), disilver disodium bis(3sulfobenzoate) heptahydrate (EKOXUY; Zheng \& Zhu, 2011), bismuth(III) 3-sulfobenzoic acid tetrahydrate (LEXKAD; Senevirathna et al., 2018), barium 3-sulfobenzoic acid trihydrate (FOBXUQ; Gao et al., 2005), and four mixed 3-sulfobenzoate hydroxo salts of the trivalent lanthanide ions neodymium (UQOYAB; Ying et al., 2010), europium (EQUBOI; Li et al., 2010), gadolinium (EQUBUO; Li et al., 2010), and terbium (EQUBIC; Li et al., 2010). All of these
Table 3

Experimental details.

\begin{tabular}{|c|c|}
\hline \multirow{2}{*}{\multicolumn{2}{|c|}{ Crystal data }} \\
\hline & \\
\hline Chemical formula & {$\left[\mathrm{Ag}\left(\mathrm{C}_{7} \mathrm{H}_{5} \mathrm{O}_{5} \mathrm{~S}\right)\right]$} \\
\hline$M_{\mathrm{r}}$ & 309.04 \\
\hline Crystal system, space group & Triclinic, $P \overline{1}$ \\
\hline Temperature $(\mathrm{K})$ & 100 \\
\hline$a, b, c(\AA)$ & $6.0376(5), 8.6293(7), 15.5903(12)$ \\
\hline$\alpha, \beta, \gamma\left({ }^{\circ}\right)$ & $92.315(1), 99.589$ (1), 90.657 (1) \\
\hline$V\left(\AA^{3}\right)$ & $800.12(11)$ \\
\hline$Z$ & 4 \\
\hline Radiation type & Мо $K \alpha$ \\
\hline$\mu\left(\mathrm{mm}^{-1}\right)$ & 2.77 \\
\hline Crystal size (mm) & $0.10 \times 0.09 \times 0.02$ \\
\hline \multicolumn{2}{|l|}{ Data collection } \\
\hline Diffractometer & Bruker APEXII CCD \\
\hline Absorption correction & $\begin{array}{l}\text { Multi-scan (SADABS; Krause et } \\
\quad \text { al., 2015) }\end{array}$ \\
\hline$T_{\min }, T_{\max }$ & $0.675,0.746$ \\
\hline $\begin{array}{l}\text { No. of measured, independent and } \\
\text { observed }[I>2 \sigma(I)] \text { reflections }\end{array}$ & $11343,3990,3464$ \\
\hline$R_{\text {int }}$ & 0.019 \\
\hline$(\sin \theta / \lambda)_{\max }\left(\AA^{-1}\right)$ & 0.668 \\
\hline \multicolumn{2}{|l|}{ Refinement } \\
\hline$R\left[F^{2}>2 \sigma\left(F^{2}\right)\right], w R\left(F^{2}\right), S$ & $0.023,0.057,1.02$ \\
\hline No. of reflections & 3990 \\
\hline No. of parameters & 255 \\
\hline $\mathrm{H}$-atom treatment & $\mathrm{H}$-atom parameters constrained \\
\hline$\Delta \rho_{\max }, \Delta \rho_{\min }\left(\mathrm{e} \AA^{-3}\right)$ & $1.46,-0.54$ \\
\hline
\end{tabular}

Computer programs: APEX3 and SAINT (Bruker, 2015), SHELXT2014/5 (Sheldrick, 2015a), SHELXL2018/3 (Sheldrick, 2015b) and CrystalMaker (Palmer, 2014).

structures feature direct bonding between the sulfonate $\mathrm{O}$ atoms and the metal ions with resulting frameworks of varying dimensionalities.

\section{Synthesis and crystallization}

A $2.24 \mathrm{~g}(10.0 \mathrm{mmol})$ sample of sodium 3-sulfobenzoic acid (Aldrich, 97\%) was dissolved in $45 \mathrm{ml}$ of water. To this colorless solution was added a colorless solution of $1.69 \mathrm{~g}$ (9.95 mmol) of $\mathrm{AgNO}_{3}$ (Baker) in $45 \mathrm{ml}$ of water. The resulting clear colorless solution was stirred for about 30 minutes and transferred to a porcelain evaporating dish that was set out to evaporate in a fume hood. After several days, the water had completely evaporated leaving behind small colorless needle-shaped crystals, $0.75 \mathrm{~g}$ of which were collected by hand from the dish. These were identified as (I) through the single crystal X-ray study.

\section{Refinement}

Crystal data, data collection and structure refinement details are summarized in Table 3. Hydrogen atoms bonded to carbon atoms and the carboxylic hydrogen atoms were located in difference electron-density maps, refined isotropically to confirm their placement, and finally, owing to the presence of the heavy atoms, constrained on idealized positions and included in the refinement as riding atoms with $\mathrm{C}-\mathrm{H}=0.95 \AA$ or $\mathrm{O}-\mathrm{H}=0.84 \AA$ and their $U_{\text {iso }}$ constrained to be $1.2(\mathrm{C}-\mathrm{H})$ or $1.5(\mathrm{O}-\mathrm{H})$ times the $U_{\text {eq }}$ of the bonding atom. There are 
four relatively large peaks $\left(1.22-1.46 \mathrm{e} \AA^{-3}\right)$ in the final difference electron-density map that are located $c a 0.9 \AA$ on either side of the $\mathrm{Ag}$ atoms along the $a$ axis. Attempted refinement of the extinction parameter resulted in a value near zero so it was not included in the final model. Although we cannot rule out an issue with the absorption correction, none is evident and the structure is otherwise well-behaved. The variable-temperature single crystal X-ray experiment was done by cooling in $10 \mathrm{~K}$ increments from 250 to $130 \mathrm{~K}$ and then heating back to $170 \mathrm{~K}$. At each step once the desired temperature was reached, the crystal was maintained at that temperature for 15 minutes before data acquisition. A complete data collection and refinement were also conducted at $296 \mathrm{~K}$ to confirm the reported monoclinic structure (Prochniak et al., 2008). Our results were essentially identical to the reported ones so they are not included here.

\section{Acknowledgements}

We thank Chris Gianopoulos (U. of Toledo) for helpful discussions on the refinement of the structure.

\section{References}

Bruker (2015). APEX3 and SAINT. Bruker AXS Inc., Madison, Wisconsin, USA.

Côté, A. P. \& Shimizu, G. K. H. (2004). Inorg. Chem. 43, 6663-6673.

Dey, C., Kundu, T., Biswal, B. P., Mallick, A. \& Banerjee, R. (2014). Acta Cryst. B70, 3-10.
Downer, S. M., Squattrito, P. J., Bestaoui, N. \& Clearfield, A. (2006). J. Chem. Crystallogr. 36, 487-501.

Gao, S., Zhu, Z.-B., Huo, L.-H. \& Ng, S. W. (2005). Acta Cryst. E61, m517-m518.

Groom, C. R., Bruno, I. J., Lightfoot, M. P. \& Ward, S. C. (2016). Acta Cryst. B72, 171-179.

Hoffart, D. J., Dalrymple, S. A. \& Shimizu, G. K. H. (2005). Inorg. Chem. 44, 8868-8875.

Krause, L., Herbst-Irmer, R., Sheldrick, G. M. \& Stalke, D. (2015). J. Appl. Cryst. 48, 3-10.

Li, X., Sun, H.-L., Wu, X.-S., Qiu, X. \& Du, M. (2010). Inorg. Chem. 49, 1865-1871.

Ma, J.-F., Yang, J. \& Liu, J.-F. (2003). Acta Cryst. E59, m478$\mathrm{m} 480$.

Palmer, D. (2014). CrystalMaker. CrystalMaker Software Ltd, Yarnton, England.

Parr, R. G. \& Pearson, R. G. (1983). J. Am. Chem. Soc. 105, 75127516.

Prochniak, G., Videnova-Adrabinska, V., Daszkiewicz, M. \& Pietraszko, A. (2008). J. Mol. Struct. 891, 178-183.

Senevirathna, D. C., Werrett, M. V., Blair, V. L., Mehring, M. \& Andrews, P. C. (2018). Chem. Eur. J. 24, 6722-6726.

Sheldrick, G. M. (2015a). Acta Cryst. A71, 3-8.

Sheldrick, G. M. (2015b). Acta Cryst. C71, 3-8.

Shimizu, G. K. H., Vaidhyanathan, R. \& Taylor, J. M. (2009). Chem. Soc. Rev. 38, 1430-1449.

Squattrito, P. J., Lambright-Mutthamsetty, K. J., Giolando, P. A. \& Kirschbaum, K. (2019). Acta Cryst. E75, 1801-1807.

Ying, S.-M., Chen, W.-T., Liu, J.-H. \& Li, X.-F. (2010). Chin. J. Struct. Chem. 29, 1547-1551.

Zheng, X.-F. \& Zhu, L.-G. (2011). Inorg. Chim. Acta, 365, 419429. 


\section{supporting information}

Acta Cryst. (2020). E76, 1275-1278 [https://doi.org/10.1107/S2056989020009408]

\section{The low-temperature triclinic crystal structure of silver 3-sulfobenzoic acid}

\section{Reuben T. Bettinger, Philip J. Squattrito and Darpandeep Aulakh}

Computing details

Data collection: APEX3 (Bruker, 2015); cell refinement: SAINT (Bruker, 2015); data reduction: SAINT (Bruker, 2015); program(s) used to solve structure: SHELXT2014/5 (Sheldrick, 2015a); program(s) used to refine structure: SHELXL2018/3 (Sheldrick, 2015b); molecular graphics: CrystalMaker (Palmer, 2014).

Poly $\left[\left(\mu_{4}-3\right.\right.$-carboxybenzenesulfonato)silver(I)]

\section{Crystal data}

$\left[\mathrm{Ag}\left(\mathrm{C}_{7} \mathrm{H}_{5} \mathrm{O}_{5} \mathrm{~S}\right)\right]$

$M_{r}=309.04$

Triclinic, $P \overline{1}$

$a=6.0376(5) \AA$

$b=8.6293(7) \AA$

$c=15.5903$ (12) $\AA$

$\alpha=92.315(1)^{\circ}$

$\beta=99.589(1)^{\circ}$

$\gamma=90.657(1)^{\circ}$

$V=800.12(11) \AA^{3}$

\section{Data collection}

Bruker APEXII CCD diffractometer

$\omega$ and $\varphi$ scans

Absorption correction: multi-scan

(SADABS; Krause et al., 2015)

$T_{\min }=0.675, T_{\max }=0.746$

11343 measured reflections

\section{Refinement}

Refinement on $F^{2}$

Least-squares matrix: full

$R\left[F^{2}>2 \sigma\left(F^{2}\right)\right]=0.023$

$w R\left(F^{2}\right)=0.057$

$S=1.02$

3990 reflections

255 parameters

0 restraints

Primary atom site location: dual
$Z=4$

$F(000)=600$

$D_{\mathrm{x}}=2.565 \mathrm{Mg} \mathrm{m}^{-3}$

Mo $K \alpha$ radiation, $\lambda=0.71073 \AA$

Cell parameters from 5814 reflections

$\theta=2.7-28.4^{\circ}$

$\mu=2.77 \mathrm{~mm}^{-1}$

$T=100 \mathrm{~K}$

Block, colorless

$0.10 \times 0.09 \times 0.02 \mathrm{~mm}$

3990 independent reflections 3464 reflections with $I>2 \sigma(I)$

$R_{\text {int }}=0.019$

$\theta_{\text {max }}=28.4^{\circ}, \theta_{\min }=2.4^{\circ}$

$h=-8 \rightarrow 8$

$k=-11 \rightarrow 11$

$l=-20 \rightarrow 20$

Secondary atom site location: difference Fourier map

Hydrogen site location: difference Fourier map

$\mathrm{H}$-atom parameters constrained

$w=1 /\left[\sigma^{2}\left(F_{\mathrm{o}}^{2}\right)+(0.0312 P)^{2}+0.7957 P\right]$

where $P=\left(F_{\mathrm{o}}^{2}+2 F_{\mathrm{c}}^{2}\right) / 3$

$(\Delta / \sigma)_{\max }=0.002$

$\Delta \rho_{\max }=1.46 \mathrm{e}^{-3}$

$\Delta \rho_{\min }=-0.54$ e $\AA^{-3}$ 


\section{Special details}

Geometry. All esds (except the esd in the dihedral angle between two 1.s. planes) are estimated using the full covariance matrix. The cell esds are taken into account individually in the estimation of esds in distances, angles and torsion angles; correlations between esds in cell parameters are only used when they are defined by crystal symmetry. An approximate (isotropic) treatment of cell esds is used for estimating esds involving l.s. planes.

Fractional atomic coordinates and isotropic or equivalent isotropic displacement parameters $\left(\hat{A}^{2}\right)$

\begin{tabular}{|c|c|c|c|c|}
\hline & $x$ & $y$ & $z$ & $U_{\text {iso }} * / U_{\text {eq }}$ \\
\hline Ag1 & $1.38401(3)$ & $0.20362(2)$ & $0.54061(2)$ & $0.01134(6)$ \\
\hline Ag2 & $0.84468(3)$ & $0.29410(2)$ & $0.45908(2)$ & $0.01133(6)$ \\
\hline S1 & $1.30827(10)$ & $0.44688(7)$ & $0.38473(4)$ & $0.00764(12)$ \\
\hline S2 & $0.91114(10)$ & $0.05323(7)$ & $0.61650(4)$ & $0.00735(12)$ \\
\hline $\mathrm{O} 1$ & $0.8095(3)$ & 0.0907 (2) & $0.04187(13)$ & $0.0171(4)$ \\
\hline $\mathrm{O} 2$ & $1.1825(3)$ & $0.1223(2)$ & $0.08315(12)$ & 0.0147 (4) \\
\hline $\mathrm{H} 2$ & 1.186373 & 0.069042 & 0.037146 & $0.022 *$ \\
\hline $\mathrm{O} 3$ & $1.4157(3)$ & $0.5928(2)$ & $0.36958(12)$ & $0.0118(4)$ \\
\hline $\mathrm{O} 4$ & $1.1960(3)$ & $0.4580(2)$ & $0.46182(12)$ & $0.0113(4)$ \\
\hline $\mathrm{O} 5$ & $1.4577(3)$ & $0.3141(2)$ & 0.38907 (12) & $0.0120(4)$ \\
\hline O6 & $1.0685(3)$ & $0.3705(2)$ & $0.92789(12)$ & $0.0151(4)$ \\
\hline $\mathrm{O} 7$ & $0.7208(3)$ & $0.4338(2)$ & 0.94908 (13) & $0.0172(4)$ \\
\hline H7 & 0.792105 & 0.496134 & 0.986827 & $0.026^{*}$ \\
\hline $\mathrm{O} 8$ & $1.0577(3)$ & $0.1870(2)$ & $0.61036(12)$ & $0.0122(4)$ \\
\hline O9 & $1.0299(3)$ & $-0.0917(2)$ & $0.63142(12)$ & $0.0113(4)$ \\
\hline $\mathrm{O} 10$ & $0.7283(3)$ & $0.0403(2)$ & $0.54050(12)$ & $0.0121(4)$ \\
\hline $\mathrm{C} 1$ & 0.9468 (4) & $0.2658(3)$ & $0.16077(16)$ & $0.0098(5)$ \\
\hline $\mathrm{C} 2$ & $1.1255(4)$ & $0.3040(3)$ & $0.22732(16)$ & $0.0089(5)$ \\
\hline $\mathrm{H} 2 \mathrm{~A}$ & 1.269323 & 0.261007 & 0.226531 & $0.011 *$ \\
\hline $\mathrm{C} 3$ & $1.0903(4)$ & $0.4059(3)$ & $0.29493(16)$ & $0.0085(5)$ \\
\hline $\mathrm{C} 4$ & 0.8808 (4) & $0.4739(3)$ & $0.29484(17)$ & $0.0098(5)$ \\
\hline H4 & 0.858595 & 0.544361 & 0.340934 & $0.012 *$ \\
\hline $\mathrm{C} 5$ & 0.7059 (4) & $0.4378(3)$ & $0.22695(17)$ & $0.0113(5)$ \\
\hline H5 & 0.564694 & 0.485685 & 0.225963 & $0.014 *$ \\
\hline C6 & $0.7358(4)$ & $0.3320(3)$ & $0.16042(17)$ & $0.0122(5)$ \\
\hline H6 & 0.614232 & 0.304894 & 0.115096 & $0.015^{*}$ \\
\hline $\mathrm{C} 7$ & $0.9731(4)$ & $0.1514(3)$ & $0.08975(17)$ & $0.0114(5)$ \\
\hline $\mathrm{C} 8$ & $0.7557(4)$ & $0.2413(3)$ & $0.84027(16)$ & $0.0101(5)$ \\
\hline C9 & $0.8758(4)$ & $0.1989(3)$ & $0.77403(16)$ & $0.0089(5)$ \\
\hline H9 & 1.022664 & 0.239791 & 0.774347 & $0.011^{*}$ \\
\hline $\mathrm{C} 10$ & 0.7759 (4) & $0.0954(3)$ & $0.70750(16)$ & $0.0083(5)$ \\
\hline C11 & $0.5641(4)$ & $0.0292(3)$ & $0.70888(16)$ & $0.0094(5)$ \\
\hline H11 & 0.498762 & -0.043294 & 0.664035 & $0.011^{*}$ \\
\hline $\mathrm{C} 12$ & $0.4502(4)$ & $0.0705(3)$ & $0.77651(17)$ & $0.0109(5)$ \\
\hline H12 & 0.307895 & 0.024014 & 0.778523 & $0.013^{*}$ \\
\hline C13 & $0.5423(4)$ & 0.1791 (3) & $0.84132(17)$ & $0.0116(5)$ \\
\hline H13 & 0.460534 & 0.210509 & 0.885891 & $0.014 *$ \\
\hline C14 & $0.8629(4)$ & $0.3547(3)$ & $0.91029(16)$ & $0.0104(5)$ \\
\hline
\end{tabular}


Atomic displacement parameters $\left(\AA^{2}\right)$

\begin{tabular}{|c|c|c|c|c|c|c|}
\hline & $U^{11}$ & $U^{22}$ & $U^{33}$ & $U^{12}$ & $U^{13}$ & $U^{23}$ \\
\hline Ag1 & $0.01230(10)$ & $0.00897(10)$ & $0.01265(10)$ & $-0.00049(7)$ & $0.00235(7)$ & $-0.00154(7)$ \\
\hline Ag2 & $0.01222(10)$ & $0.00908(10)$ & $0.01249(10)$ & $0.00046(7)$ & $0.00190(7)$ & $-0.00134(7)$ \\
\hline $\mathrm{S} 1$ & $0.0092(3)$ & 0.0059 (3) & $0.0076(3)$ & $0.0002(2)$ & $0.0012(2)$ & $-0.0016(2)$ \\
\hline $\mathrm{S} 2$ & $0.0092(3)$ & 0.0058 & 0.0071 & $-0.0001(2)$ & $0.0017(2)$ & $-0.0015(2)$ \\
\hline $\mathrm{O} 1$ & $0.0147(10)$ & $0.0189(10)$ & $0.0160(10)$ & $0.0011(7)$ & $0.0001(8)$ & $-0.0103(8)$ \\
\hline $\mathrm{O} 2$ & $0.0149(9)$ & $0.0163(10)$ & $0.0122(9)$ & $0.0022(7)$ & $0.0015(7)$ & $-0.0069(7)$ \\
\hline $\mathrm{O} 3$ & $0.0131(9)$ & $0.0091(9)$ & $0.0124(9)$ & $-0.0021(7)$ & $-0.0005(7)$ & $0.0006(7)$ \\
\hline $\mathrm{O} 4$ & $0.0133(9)$ & $0.0128(9)$ & $0.0079(8)$ & $-0.0011(7)$ & $0.0025(7)$ & $-0.0014(7)$ \\
\hline $\mathrm{O} 5$ & $0.0121(9)$ & $0.0079(8)$ & $0.0151(9)$ & $0.0026(7)$ & $-0.0003(7)$ & $-0.0016(7)$ \\
\hline O6 & $0.0154(10)$ & $0.0156(10)$ & $0.0134(9)$ & $-0.0029(7)$ & $0.0011(7)$ & $-0.0046(7)$ \\
\hline $\mathrm{O} 7$ & $0.0188(10)$ & $0.0173(10)$ & $0.0150(10)$ & $-0.0009(8)$ & $0.0041(8)$ & $-0.0104(8)$ \\
\hline O8 & $0.0161(9)$ & $0.0067(8)$ & $0.0151(9)$ & $-0.0025(7)$ & $0.0070(7)$ & $-0.0017(7)$ \\
\hline O9 & $0.0132(9)$ & $0.0087(8)$ & $0.0128(9)$ & $0.0037(7)$ & $0.0049(7)$ & $-0.0006(7)$ \\
\hline $\mathrm{O} 10$ & $0.0126(9)$ & $0.0138(9)$ & $0.0090(9)$ & $0.0011(7)$ & $-0.0004(7)$ & $-0.0025(7)$ \\
\hline $\mathrm{C} 1$ & $0.0139(12)$ & $0.0069(11)$ & $0.0087(11)$ & $0.0001(9)$ & $0.0025(9)$ & $-0.0011(9)$ \\
\hline $\mathrm{C} 2$ & $0.0098(12)$ & $0.0071(11)$ & $0.0098(11)$ & $0.0011(9)$ & $0.0016(9)$ & $0.0001(9)$ \\
\hline $\mathrm{C} 3$ & $0.0093(11)$ & $0.0087(11)$ & $0.0071(11)$ & $-0.0002(9)$ & $0.0005(9)$ & $0.0006(9)$ \\
\hline $\mathrm{C} 4$ & $0.0124(12)$ & 0.0075 (11) & $0.0096(11)$ & $-0.0002(9)$ & $0.0023(9)$ & $-0.0010(9)$ \\
\hline $\mathrm{C} 5$ & $0.0097(12)$ & $0.0105(12)$ & $0.0143(12)$ & $0.0020(9)$ & $0.0034(9)$ & $0.0018(10)$ \\
\hline C6 & $0.0125(12)$ & $0.0112(12)$ & 0.0119 (12) & $-0.0018(9)$ & $-0.0004(9)$ & $0.0003(9)$ \\
\hline $\mathrm{C} 7$ & $0.0170(13)$ & $0.0092(11)$ & 0.0083 (12) & $0.0014(9)$ & $0.0023(9)$ & $0.0011(9)$ \\
\hline $\mathrm{C} 8$ & $0.0127(12)$ & $0.0082(11)$ & $0.0087(12)$ & 0.0007 (9) & $0.0000(9)$ & $-0.0002(9)$ \\
\hline C9 & $0.0087(11)$ & $0.0064(11)$ & 0.0112 (12) & $-0.0002(9)$ & $0.0004(9)$ & $0.0012(9)$ \\
\hline $\mathrm{C} 10$ & $0.0097(12)$ & $0.0080(11)$ & $0.0072(11)$ & $0.0019(9)$ & $0.0018(9)$ & $-0.0015(9)$ \\
\hline C11 & $0.0117(12)$ & $0.0069(11)$ & $0.0091(11)$ & $0.0006(9)$ & $0.0005(9)$ & $0.0003(9)$ \\
\hline C12 & $0.0082(11)$ & $0.0112(12)$ & 0.0133 (12) & $-0.0001(9)$ & $0.0020(9)$ & $0.0002(9)$ \\
\hline C13 & $0.0120(12)$ & $0.0131(12)$ & 0.0097 (12) & $0.0013(9)$ & $0.0021(9)$ & $-0.0006(9)$ \\
\hline $\mathrm{C} 14$ & $0.0161(13)$ & $0.0093(11)$ & $0.0061(11)$ & $0.0004(9)$ & $0.0026(9)$ & $0.0004(9)$ \\
\hline
\end{tabular}

Geometric parameters $\left(A,{ }^{\circ}\right)$

\begin{tabular}{|c|c|c|c|}
\hline $\mathrm{Ag} 1-\mathrm{O} 3^{\mathrm{i}}$ & 2.3868 (18) & $\mathrm{O} 7-\mathrm{C} 14$ & $1.311(3)$ \\
\hline $\mathrm{Ag} 1-\mathrm{O} 8$ & $2.4091(18)$ & $\mathrm{O} 7-\mathrm{H} 7$ & 0.8400 \\
\hline $\mathrm{Ag} 1-\mathrm{O} 10^{\mathrm{ii}}$ & $2.4406(18)$ & $\mathrm{C} 1-\mathrm{C} 2$ & $1.393(3)$ \\
\hline Ag1-O10 & $2.5249(18)$ & $\mathrm{C} 1-\mathrm{C} 6$ & $1.402(4)$ \\
\hline $\mathrm{Ag} 1-\mathrm{O} 5$ & 2.6853 (19) & $\mathrm{C} 1-\mathrm{C} 7$ & $1.483(3)$ \\
\hline $\mathrm{Ag} 1-\mathrm{O} 4$ & $2.7254(19)$ & $\mathrm{C} 2-\mathrm{C} 3$ & $1.390(3)$ \\
\hline $\mathrm{Ag} 2-\mathrm{O} 9^{\mathrm{ii}}$ & $2.4090(17)$ & $\mathrm{C} 2-\mathrm{H} 2 \mathrm{~A}$ & 0.9500 \\
\hline $\mathrm{Ag} 2-\mathrm{O} 5^{\mathrm{iv}}$ & $2.4199(18)$ & $\mathrm{C} 3-\mathrm{C} 4$ & $1.400(3)$ \\
\hline $\mathrm{Ag} 2-\mathrm{O} 4^{\mathrm{v}}$ & 2.4609 (18) & $\mathrm{C} 4-\mathrm{C} 5$ & $1.388(4)$ \\
\hline $\mathrm{Ag} 2-\mathrm{O} 4$ & $2.5295(18)$ & $\mathrm{C} 4-\mathrm{H} 4$ & 0.9500 \\
\hline $\mathrm{Ag} 2-\mathrm{O} 8$ & 2.6953 (19) & $\mathrm{C} 5-\mathrm{C} 6$ & $1.389(4)$ \\
\hline $\operatorname{Ag} 2-\mathrm{O} 10$ & 2.7179 (19) & $\mathrm{C} 5-\mathrm{H} 5$ & 0.9500 \\
\hline $\mathrm{S} 1-\mathrm{O} 3$ & $1.4556(18)$ & C6- $-\mathrm{H} 6$ & 0.9500 \\
\hline $\mathrm{S} 1-\mathrm{O} 5$ & $1.4629(18)$ & $\mathrm{C} 8-\mathrm{C} 13$ & $1.393(4)$ \\
\hline
\end{tabular}




\begin{tabular}{|c|c|c|c|}
\hline $\mathrm{S} 1-\mathrm{O} 4$ & $1.4754(18)$ & $\mathrm{C} 8-\mathrm{C} 9$ & $1.397(3)$ \\
\hline $\mathrm{S} 1-\mathrm{C} 3$ & $1.776(3)$ & $\mathrm{C} 8-\mathrm{C} 14$ & $1.494(3)$ \\
\hline $\mathrm{S} 2-\mathrm{O} 9$ & $1.4560(18)$ & $\mathrm{C} 9-\mathrm{C} 10$ & $1.393(3)$ \\
\hline $\mathrm{S} 2-\mathrm{O} 8$ & $1.4624(18)$ & $\mathrm{C} 9-\mathrm{H} 9$ & 0.9500 \\
\hline $\mathrm{S} 2-\mathrm{O} 10$ & $1.4784(19)$ & $\mathrm{C} 10-\mathrm{C} 11$ & $1.399(3)$ \\
\hline $\mathrm{S} 2-\mathrm{C} 10$ & $1.778(2)$ & $\mathrm{C} 11-\mathrm{C} 12$ & $1.388(3)$ \\
\hline $\mathrm{O} 1-\mathrm{C} 7$ & $1.232(3)$ & $\mathrm{C} 11-\mathrm{H} 11$ & 0.9500 \\
\hline $\mathrm{O} 2-\mathrm{C} 7$ & $1.312(3)$ & $\mathrm{C} 12-\mathrm{C} 13$ & $1.390(4)$ \\
\hline $\mathrm{O} 2-\mathrm{H} 2$ & 0.8400 & $\mathrm{C} 12-\mathrm{H} 12$ & 0.9500 \\
\hline $\mathrm{O} 6-\mathrm{C} 14$ & $1.231(3)$ & $\mathrm{C} 13-\mathrm{H} 13$ & 0.9500 \\
\hline $\mathrm{O} 3^{\mathrm{i}}-\mathrm{Ag} 1-\mathrm{O} 8$ & $99.00(6)$ & $\mathrm{Ag} 1^{\mathrm{i}}-\mathrm{O} 3-\mathrm{Ag} 2^{\mathrm{v}}$ & $67.35(4)$ \\
\hline $\mathrm{O} 3^{\mathrm{i}}-\mathrm{Ag} 1-\mathrm{O} 10^{\mathrm{ii}}$ & $164.88(6)$ & $\mathrm{S} 1-\mathrm{O} 3-\mathrm{Ag} 2^{\mathrm{iii}}$ & $68.22(7)$ \\
\hline $\mathrm{O} 8-\mathrm{Ag} 1-\mathrm{O} 10^{\mathrm{ii}}$ & $89.93(6)$ & $\mathrm{Ag} 1^{\mathrm{i}}-\mathrm{O} 3-\mathrm{Ag} 2^{\mathrm{iii}}$ & $91.87(5)$ \\
\hline $\mathrm{O} 3^{\mathrm{i}}-\mathrm{Ag} 1-\mathrm{O} 10^{\mathrm{iii}}$ & $93.71(6)$ & $\mathrm{Ag} 2^{\mathrm{v}}-\mathrm{O} 3-\mathrm{Ag} 2^{\mathrm{iii}}$ & $105.51(5)$ \\
\hline $\mathrm{O} 8-\mathrm{Ag} 1-\mathrm{O} 10^{\mathrm{iii}}$ & $134.17(6)$ & $\mathrm{S} 1-\mathrm{O} 4-\mathrm{Ag} 2^{\mathrm{v}}$ & $122.51(10)$ \\
\hline $\mathrm{O} 10^{\mathrm{ii}}-\mathrm{Ag} 1-\mathrm{O} 10^{\mathrm{iii}}$ & $71.42(7)$ & $\mathrm{S} 1-\mathrm{O} 4-\mathrm{Ag} 2$ & $117.62(10)$ \\
\hline $\mathrm{O} 3^{\mathrm{i}-\mathrm{Ag} 1-\mathrm{O} 5}$ & $95.65(6)$ & $\mathrm{Ag} 2^{\mathrm{v}}-\mathrm{O} 4-\mathrm{Ag} 2$ & $108.75(7)$ \\
\hline $\mathrm{O} 8-\mathrm{Ag} 1-\mathrm{O} 5$ & $133.72(6)$ & $\mathrm{S} 1-\mathrm{O} 4-\mathrm{Ag} 1$ & $97.01(9)$ \\
\hline $\mathrm{O} 10^{\mathrm{ii}}-\mathrm{Ag} 1-\mathrm{O} 5$ & $86.75(6)$ & $\mathrm{Ag} 2^{\mathrm{v}}-\mathrm{O} 4-\mathrm{Ag} 1$ & $123.21(7)$ \\
\hline $\mathrm{O} 10^{\mathrm{iii}-\mathrm{Ag} 1-\mathrm{O} 5}$ & $87.86(6)$ & $\mathrm{Ag} 2-\mathrm{O} 4-\mathrm{Ag} 1$ & $80.66(5)$ \\
\hline $\mathrm{O} 3^{\mathrm{i}}-\mathrm{Ag} 1-\mathrm{O} 4$ & $79.09(6)$ & $\mathrm{S} 1-\mathrm{O} 4-\mathrm{Ag} 1^{\mathrm{i}}$ & $68.13(7)$ \\
\hline $\mathrm{O} 8-\mathrm{Ag} 1-\mathrm{O} 4$ & $87.10(6)$ & $\mathrm{Ag} 2^{\mathrm{v}}-\mathrm{O} 4-\mathrm{Ag} 1^{\mathrm{i}}$ & $59.17(4)$ \\
\hline $\mathrm{O} 10^{\mathrm{ii}}-\mathrm{Ag} 1-\mathrm{O} 4$ & $113.75(6)$ & $\mathrm{Ag} 2-\mathrm{O} 4-\mathrm{Ag} 1^{\mathrm{i}}$ & $164.54(7)$ \\
\hline 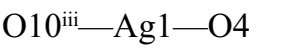 & $138.66(6)$ & $\mathrm{Ag} 1-\mathrm{O} 4-\mathrm{Ag} 1^{\mathrm{i}}$ & $113.67(5)$ \\
\hline $\mathrm{O} 5-\mathrm{Ag} 1-\mathrm{O} 4$ & $53.11(5)$ & $\mathrm{S} 1-\mathrm{O} 5-\mathrm{Ag} 2^{\mathrm{iii}}$ & $129.94(11)$ \\
\hline $\mathrm{O} 9^{\mathrm{ii}}-\mathrm{Ag} 2-\mathrm{O}^{\mathrm{iv}}$ & $100.47(6)$ & $\mathrm{S} 1-\mathrm{O} 5-\mathrm{Ag} 1$ & $99.04(9)$ \\
\hline $\mathrm{O} 9^{\mathrm{ii}}-\mathrm{Ag} 2-\mathrm{O}^{\mathrm{v}}$ & $163.71(6)$ & 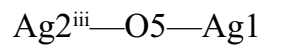 & $81.60(5)$ \\
\hline $\mathrm{O}^{5 \mathrm{iv}}-\mathrm{Ag} 2-\mathrm{O} 4^{\mathrm{v}}$ & $88.30(6)$ & $\mathrm{C} 14-\mathrm{O} 7-\mathrm{H} 7$ & 109.5 \\
\hline $\mathrm{O} 9^{\mathrm{ii}}-\mathrm{Ag} 2-\mathrm{O} 4$ & $92.99(6)$ & $\mathrm{S} 2-\mathrm{O} 8-\mathrm{Ag} 1$ & $129.24(10)$ \\
\hline $\mathrm{O} 5^{\mathrm{iv}}-\mathrm{Ag} 2-\mathrm{O} 4$ & $134.11(6)$ & $\mathrm{S} 2-\mathrm{O} 8-\mathrm{Ag} 2$ & $98.80(9)$ \\
\hline 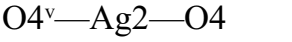 & $71.25(7)$ & Ag1-O8-Ag2 & $83.46(6)$ \\
\hline $\mathrm{O} 9^{\mathrm{ii}}-\mathrm{Ag} 2-\mathrm{O} 8$ & $95.18(6)$ & $\mathrm{S} 2-\mathrm{O} 9-\mathrm{Ag} 2^{\mathrm{ii}}$ & $135.66(11)$ \\
\hline $\mathrm{O} 5^{\mathrm{iv}}-\mathrm{Ag} 2-\mathrm{O} 8$ & $135.75(6)$ & $\mathrm{S} 2-\mathrm{O} 9-\mathrm{Ag} 1^{\mathrm{ii}}$ & $79.15(8)$ \\
\hline $\mathrm{O} 4 \mathrm{v}-\mathrm{Ag} 2-\mathrm{O} 8$ & $87.79(6)$ & $\mathrm{Ag} 2^{\mathrm{ii}}-\mathrm{O} 9-\mathrm{Ag} 1^{\mathrm{ii}}$ & $68.49(4)$ \\
\hline $\mathrm{O} 4-\mathrm{Ag} 2-\mathrm{O} 8$ & $85.39(6)$ & $\mathrm{S} 2-\mathrm{O} 9-\mathrm{Ag} 1$ & $68.85(7)$ \\
\hline $\mathrm{O} 9^{\mathrm{ii}}-\mathrm{Ag} 2-\mathrm{O} 10$ & $79.91(6)$ & $\mathrm{Ag} 22^{\mathrm{ii}}-\mathrm{O} 9-\mathrm{Ag} 1$ & $90.55(5)$ \\
\hline $\mathrm{O} 5^{\mathrm{iv}}-\mathrm{Ag} 2-\mathrm{O} 10$ & $89.29(6)$ & $\mathrm{Ag} 1{ }^{\mathrm{ii}}-\mathrm{O} 9-\mathrm{Ag} 1$ & $104.81(5)$ \\
\hline $\mathrm{O} 4^{\mathrm{v}}-\mathrm{Ag} 2-\mathrm{O} 10$ & $114.18(6)$ & $\mathrm{S} 2-\mathrm{O} 10-\mathrm{Ag} 1^{\mathrm{ii}}$ & $123.30(10)$ \\
\hline $\mathrm{O} 4-\mathrm{Ag} 2-\mathrm{O} 10$ & $136.43(6)$ & $\mathrm{S} 2-\mathrm{O} 10-\mathrm{Ag} 1^{\mathrm{iv}}$ & $118.98(10)$ \\
\hline $\mathrm{O} 8-\mathrm{Ag} 2-\mathrm{O} 10$ & $53.09(5)$ & $\mathrm{Ag} 1^{\mathrm{ii}}-\mathrm{O} 10-\mathrm{Ag} 1^{\mathrm{iv}}$ & $108.58(7)$ \\
\hline $\mathrm{O} 3-\mathrm{S} 1-\mathrm{O} 5$ & $113.96(11)$ & $\mathrm{S} 2-\mathrm{O} 10-\mathrm{Ag} 2$ & $97.40(9)$ \\
\hline $\mathrm{O} 3-\mathrm{S} 1-\mathrm{O} 4$ & $112.15(11)$ & $\mathrm{Ag} 11^{\mathrm{ii}}-\mathrm{O} 10-\mathrm{Ag} 2$ & $121.23(7)$ \\
\hline $\mathrm{O} 5-\mathrm{S} 1-\mathrm{O} 4$ & $110.84(11)$ & $\mathrm{Ag} 1^{\mathrm{iv}}-\mathrm{O} 10-\mathrm{Ag} 2$ & $79.11(5)$ \\
\hline $\mathrm{O} 3-\mathrm{S} 1-\mathrm{C} 3$ & $107.55(11)$ & $\mathrm{S} 2-\mathrm{O} 10-\mathrm{Ag} 2^{\mathrm{ii}}$ & $67.58(7)$ \\
\hline $\mathrm{O} 5-\mathrm{S} 1-\mathrm{C} 3$ & $106.29(11)$ & $\mathrm{Ag} 1^{\mathrm{ii}}-\mathrm{O} 10-\mathrm{Ag} 2^{\mathrm{ii}}$ & $59.96(4)$ \\
\hline $\mathrm{O} 4-\mathrm{S} 1-\mathrm{C} 3$ & $105.45(11)$ & $\mathrm{Ag} 1^{\mathrm{iv}}-\mathrm{O} 10-\mathrm{Ag} 2^{\mathrm{ii}}$ & $166.05(7)$ \\
\hline $\mathrm{O} 3-\mathrm{S} 1-\mathrm{Ag} 1$ & $134.18(8)$ & $\mathrm{Ag} 2-\mathrm{O} 10-\mathrm{Ag} 2^{\mathrm{ii}}$ & $113.18(5)$ \\
\hline
\end{tabular}




\begin{tabular}{|c|c|c|c|}
\hline $\mathrm{O} 5-\mathrm{S} 1-\mathrm{Ag} 1$ & $54.60(8)$ & $\mathrm{C} 2-\mathrm{C} 1-\mathrm{C} 6$ & $120.7(2)$ \\
\hline $\mathrm{O} 4-\mathrm{S} 1-\mathrm{Ag} 1$ & $56.24(7)$ & $\mathrm{C} 2-\mathrm{C} 1-\mathrm{C} 7$ & $121.0(2)$ \\
\hline $\mathrm{C} 3-\mathrm{S} 1-\mathrm{Ag} 1$ & $118.27(8)$ & $\mathrm{C} 6-\mathrm{C} 1-\mathrm{C} 7$ & $118.3(2)$ \\
\hline $\mathrm{O} 3-\mathrm{S} 1-\mathrm{Ag} 2$ & $142.49(8)$ & $\mathrm{C} 3-\mathrm{C} 2-\mathrm{C} 1$ & $119.0(2)$ \\
\hline $\mathrm{O} 5-\mathrm{S} 1-\mathrm{Ag} 2$ & $101.87(8)$ & $\mathrm{C} 3-\mathrm{C} 2-\mathrm{H} 2 \mathrm{~A}$ & 120.5 \\
\hline $\mathrm{C} 3-\mathrm{S} 1-\mathrm{Ag} 2$ & $70.67(8)$ & $\mathrm{C} 1-\mathrm{C} 2-\mathrm{H} 2 \mathrm{~A}$ & 120.5 \\
\hline $\mathrm{Ag} 1-\mathrm{S} 1-\mathrm{Ag} 2$ & $60.743(12)$ & $\mathrm{C} 2-\mathrm{C} 3-\mathrm{C} 4$ & $120.7(2)$ \\
\hline $\mathrm{O} 3-\mathrm{S} 1-\mathrm{Ag} 2^{\mathrm{v}}$ & $75.89(8)$ & $\mathrm{C} 2-\mathrm{C} 3-\mathrm{S} 1$ & $120.38(19)$ \\
\hline $\mathrm{O} 5-\mathrm{S} 1-\mathrm{Ag} 2^{\mathrm{v}}$ & $133.57(8)$ & $\mathrm{C} 4-\mathrm{C} 3-\mathrm{S} 1$ & $118.86(19)$ \\
\hline $\mathrm{C} 3-\mathrm{S} 1-\mathrm{Ag} 2^{\mathrm{v}}$ & $113.59(8)$ & $\mathrm{C} 2-\mathrm{C} 3-\mathrm{Ag} 2$ & $122.32(16)$ \\
\hline $\mathrm{Ag} 1-\mathrm{S} 1-\mathrm{Ag} 2^{\mathrm{v}}$ & $85.217(16)$ & $\mathrm{C} 4-\mathrm{C} 3-\mathrm{Ag} 2$ & $67.59(14)$ \\
\hline $\mathrm{Ag} 2-\mathrm{S} 1-\mathrm{Ag} 2^{\mathrm{v}}$ & $71.389(13)$ & $\mathrm{S} 1-\mathrm{C} 3-\mathrm{Ag} 2$ & $79.16(8)$ \\
\hline $\mathrm{O} 3-\mathrm{S} 1-\mathrm{Ag} 2^{\mathrm{iii}}$ & $89.34(8)$ & $\mathrm{C} 5-\mathrm{C} 4-\mathrm{C} 3$ & $119.5(2)$ \\
\hline $\mathrm{O} 4-\mathrm{S} 1-\mathrm{Ag} 2^{\mathrm{iii}}$ & $105.55(8)$ & $\mathrm{C} 5-\mathrm{C} 4-\mathrm{Ag} 2$ & $112.49(17)$ \\
\hline $\mathrm{C} 3-\mathrm{S} 1-\mathrm{Ag} 2^{\mathrm{iii}}$ & $135.36(8)$ & $\mathrm{C} 3-\mathrm{C} 4-\mathrm{Ag} 2$ & $87.60(15)$ \\
\hline $\mathrm{Ag} 1-\mathrm{S} 1-\mathrm{Ag} 2^{\mathrm{iii}}$ & $58.742(11)$ & $\mathrm{C} 5-\mathrm{C} 4-\mathrm{H} 4$ & 120.2 \\
\hline $\mathrm{Ag} 2-\mathrm{S} 1-\mathrm{Ag} 2^{\mathrm{iii}}$ & $118.903(18)$ & $\mathrm{C} 3-\mathrm{C} 4-\mathrm{H} 4$ & 120.2 \\
\hline $\mathrm{Ag} 2^{\mathrm{v}}-\mathrm{S} 1-\mathrm{Ag} 2^{\mathrm{iii}}$ & $110.509(17)$ & $\mathrm{Ag} 2-\mathrm{C} 4-\mathrm{H} 4$ & 70.2 \\
\hline $\mathrm{O} 5-\mathrm{S} 1-\mathrm{Ag} 1^{\mathrm{i}}$ & $110.81(8)$ & $\mathrm{C} 4-\mathrm{C} 5-\mathrm{C} 6$ & $120.5(2)$ \\
\hline $\mathrm{O} 4-\mathrm{S} 1-\mathrm{Ag} 1^{\mathrm{i}}$ & $89.35(7)$ & $\mathrm{C} 4-\mathrm{C} 5-\mathrm{Ag} 2$ & $47.98(13)$ \\
\hline $\mathrm{C} 3-\mathrm{S} 1-\mathrm{Ag} 1^{\mathrm{i}}$ & $131.60(8)$ & $\mathrm{C} 6-\mathrm{C} 5-\mathrm{Ag} 2$ & $116.08(17)$ \\
\hline $\mathrm{Ag} 1-\mathrm{S} 1-\mathrm{Ag} 1^{\mathrm{i}}$ & $108.357(17)$ & $\mathrm{C} 4-\mathrm{C} 5-\mathrm{H} 5$ & 119.7 \\
\hline $\mathrm{Ag} 2-\mathrm{S} 1-\mathrm{Ag} 1^{\mathrm{i}}$ & 127.793 (18) & $\mathrm{C} 6-\mathrm{C} 5-\mathrm{H} 5$ & 119.7 \\
\hline $\mathrm{Ag} 2^{\mathrm{v}}-\mathrm{S} 1-\mathrm{Ag} 1^{\mathrm{i}}$ & $56.515(11)$ & $\mathrm{Ag} 2-\mathrm{C} 5-\mathrm{H} 5$ & 103.4 \\
\hline $\mathrm{Ag} 2^{\mathrm{iii}}-\mathrm{S} 1-\mathrm{Ag} 1^{\mathrm{i}}$ & $79.804(14)$ & $\mathrm{C} 5-\mathrm{C} 6-\mathrm{C} 1$ & $119.4(2)$ \\
\hline $\mathrm{O} 9-\mathrm{S} 2-\mathrm{O} 8$ & $114.02(11)$ & $\mathrm{C} 5-\mathrm{C} 6-\mathrm{H} 6$ & 120.3 \\
\hline $\mathrm{O} 9-\mathrm{S} 2-\mathrm{O} 10$ & $112.31(11)$ & $\mathrm{C} 1-\mathrm{C} 6-\mathrm{H} 6$ & 120.3 \\
\hline $\mathrm{O} 8-\mathrm{S} 2-\mathrm{O} 10$ & $110.71(11)$ & $\mathrm{O} 1-\mathrm{C} 7-\mathrm{O} 2$ & $124.1(2)$ \\
\hline $\mathrm{O} 9-\mathrm{S} 2-\mathrm{C} 10$ & $107.87(11)$ & $\mathrm{O} 1-\mathrm{C} 7-\mathrm{C} 1$ & $121.7(2)$ \\
\hline $\mathrm{O} 8-\mathrm{S} 2-\mathrm{C} 10$ & $106.02(11)$ & $\mathrm{O} 2-\mathrm{C} 7-\mathrm{C} 1$ & $114.2(2)$ \\
\hline $\mathrm{O} 10-\mathrm{S} 2-\mathrm{C} 10$ & $105.29(11)$ & $\mathrm{C} 13-\mathrm{C} 8-\mathrm{C} 9$ & $120.9(2)$ \\
\hline $\mathrm{O} 9-\mathrm{S} 2-\mathrm{Ag} 2$ & $134.36(8)$ & $\mathrm{C} 13-\mathrm{C} 8-\mathrm{C} 14$ & $120.9(2)$ \\
\hline $\mathrm{O} 8-\mathrm{S} 2-\mathrm{Ag} 2$ & $54.86(8)$ & $\mathrm{C} 9-\mathrm{C} 8-\mathrm{C} 14$ & $118.2(2)$ \\
\hline $\mathrm{O} 10-\mathrm{S} 2-\mathrm{Ag} 2$ & $55.85(8)$ & $\mathrm{C} 10-\mathrm{C} 9-\mathrm{C} 8$ & $118.7(2)$ \\
\hline $\mathrm{C} 10-\mathrm{S} 2-\mathrm{Ag} 2$ & $117.76(8)$ & $\mathrm{C} 10-\mathrm{C} 9-\mathrm{H} 9$ & 120.6 \\
\hline $\mathrm{O} 9-\mathrm{S} 2-\mathrm{Ag} 1^{\mathrm{ii}}$ & $76.58(8)$ & $\mathrm{C} 8-\mathrm{C} 9-\mathrm{H} 9$ & 120.6 \\
\hline $\mathrm{O} 8-\mathrm{S} 2-\mathrm{Ag} 1^{\mathrm{ii}}$ & $132.19(8)$ & $\mathrm{C} 9-\mathrm{C} 10-\mathrm{C} 11$ & $120.9(2)$ \\
\hline $\mathrm{C} 10-\mathrm{S} 2-\mathrm{Ag} 1^{\mathrm{ii}}$ & $114.74(8)$ & $\mathrm{C} 9-\mathrm{C} 10-\mathrm{S} 2$ & $120.11(19)$ \\
\hline $\mathrm{Ag} 2-\mathrm{S} 2-\mathrm{Ag} 1^{\mathrm{ii}}$ & $83.690(15)$ & $\mathrm{C} 11-\mathrm{C} 10-\mathrm{S} 2$ & $118.95(19)$ \\
\hline $\mathrm{O} 9-\mathrm{S} 2-\mathrm{Ag} 1^{\mathrm{iv}}$ & $142.72(8)$ & $\mathrm{C} 9-\mathrm{C} 10-\mathrm{Ag} 1^{\mathrm{iv}}$ & $122.76(16)$ \\
\hline $\mathrm{O} 8-\mathrm{S} 2-\mathrm{Ag} 1^{\mathrm{iv}}$ & $101.22(8)$ & $\mathrm{C} 11-\mathrm{C} 10-\mathrm{Ag} 1^{\mathrm{iv}}$ & $67.27(14)$ \\
\hline $\mathrm{C} 10-\mathrm{S} 2-\mathrm{Ag} 1^{\mathrm{iv}}$ & $71.67(8)$ & $\mathrm{S} 2-\mathrm{C} 10-\mathrm{Ag} 1^{\mathrm{iv}}$ & $78.38(8)$ \\
\hline $\mathrm{Ag} 2-\mathrm{S} 2-\mathrm{Ag} 1^{\mathrm{iv}}$ & $59.268(11)$ & $\mathrm{C} 12-\mathrm{C} 11-\mathrm{C} 10$ & $119.3(2)$ \\
\hline $\mathrm{Ag} 1^{1 \mathrm{ii}}-\mathrm{S} 2-\mathrm{Ag} 1^{\mathrm{iv}}$ & $70.705(13)$ & $\mathrm{C} 12-\mathrm{C} 11-\mathrm{Ag} 1^{\mathrm{iv}}$ & $111.71(16)$ \\
\hline $\mathrm{O} 9-\mathrm{S} 2-\mathrm{Ag} 1$ & $88.47(8)$ & $\mathrm{C} 10-\mathrm{C} 11-\mathrm{Ag} 1^{\mathrm{iv}}$ & $88.31(15)$ \\
\hline $\mathrm{O} 10-\mathrm{S} 2-\mathrm{Ag} 1$ & $106.42(8)$ & $\mathrm{C} 12-\mathrm{C} 11-\mathrm{H} 11$ & 120.4 \\
\hline $\mathrm{C} 10-\mathrm{S} 2-\mathrm{Ag} 1$ & $135.01(8)$ & $\mathrm{C} 10-\mathrm{C} 11-\mathrm{H} 11$ & 120.4 \\
\hline $\mathrm{Ag} 2-\mathrm{S} 2-\mathrm{Ag} 1$ & $60.142(11)$ & $\mathrm{Ag} 1^{\mathrm{iv}}-\mathrm{C} 11-\mathrm{H} 11$ & 70.3 \\
\hline
\end{tabular}




\begin{tabular}{|c|c|c|c|}
\hline $\mathrm{Ag} 1{ }^{\mathrm{ii}}-\mathrm{S} 2-\mathrm{Ag} 1$ & 109.706 (17) & $\mathrm{C} 11-\mathrm{C} 12-\mathrm{C} 13$ & $120.7(2)$ \\
\hline $\mathrm{Ag} 1^{\mathrm{iv}}-\mathrm{S} 2-\mathrm{Ag} 1$ & $118.896(18)$ & $\mathrm{C} 11-\mathrm{C} 12-\mathrm{Ag} 1^{\mathrm{iv}}$ & $48.75(13)$ \\
\hline $\mathrm{O} 8-\mathrm{S} 2-\mathrm{Ag} 2^{\mathrm{ii}}$ & $109.90(8)$ & $\mathrm{C} 13-\mathrm{C} 12-\mathrm{Ag} 1^{\mathrm{iv}}$ & $116.17(17)$ \\
\hline $\mathrm{O} 10-\mathrm{S} 2-\mathrm{Ag} 2^{\mathrm{ii}}$ & $90.09(7)$ & $\mathrm{C} 11-\mathrm{C} 12-\mathrm{H} 12$ & 119.6 \\
\hline $\mathrm{C} 10-\mathrm{S} 2-\mathrm{Ag} 2^{\mathrm{ii}}$ & $132.48(8)$ & $\mathrm{C} 13-\mathrm{C} 12-\mathrm{H} 12$ & 119.6 \\
\hline $\mathrm{Ag} 2-\mathrm{S} 2-\mathrm{Ag} 2^{\mathrm{ii}}$ & $108.167(17)$ & $\mathrm{Ag} 1^{\mathrm{iv}}-\mathrm{C} 12-\mathrm{H} 12$ & 102.7 \\
\hline $\mathrm{Ag} 1^{\mathrm{ii}}-\mathrm{S} 2-\mathrm{Ag} 2^{\mathrm{ii}}$ & $57.481(11)$ & $\mathrm{C} 12-\mathrm{C} 13-\mathrm{C} 8$ & $119.4(2)$ \\
\hline $\mathrm{Ag} 1^{\mathrm{iv}}-\mathrm{S} 2-\mathrm{Ag} 2^{\mathrm{ii}}$ & $128.036(18)$ & $\mathrm{C} 12-\mathrm{C} 13-\mathrm{H} 13$ & 120.3 \\
\hline $\mathrm{Ag} 1-\mathrm{S} 2-\mathrm{Ag} 2^{\mathrm{ii}}$ & $78.344(13)$ & $\mathrm{C} 8-\mathrm{C} 13-\mathrm{H} 13$ & 120.3 \\
\hline $\mathrm{C} 7-\mathrm{O} 2-\mathrm{H} 2$ & 109.5 & $\mathrm{O} 6-\mathrm{C} 14-\mathrm{O} 7$ & $124.4(2)$ \\
\hline $\mathrm{S} 1-\mathrm{O} 3-\mathrm{Ag} 1^{\mathrm{i}}$ & $135.48(11)$ & $\mathrm{O} 6-\mathrm{C} 14-\mathrm{C} 8$ & $121.1(2)$ \\
\hline $\mathrm{S} 1-\mathrm{O} 3-\mathrm{Ag}^{\mathrm{v}}$ & $79.83(8)$ & $\mathrm{O} 7-\mathrm{C} 14-\mathrm{C} 8$ & $114.6(2)$ \\
\hline $\mathrm{C} 6-\mathrm{C} 1-\mathrm{C} 2-\mathrm{C} 3$ & $1.7(4)$ & $\mathrm{C} 13-\mathrm{C} 8-\mathrm{C} 9-\mathrm{C} 10$ & $1.5(4)$ \\
\hline $\mathrm{C} 7-\mathrm{C} 1-\mathrm{C} 2-\mathrm{C} 3$ & $-176.8(2)$ & $\mathrm{C} 14-\mathrm{C} 8-\mathrm{C} 9-\mathrm{C} 10$ & $-178.8(2)$ \\
\hline $\mathrm{C} 1-\mathrm{C} 2-\mathrm{C} 3-\mathrm{C} 4$ & $-2.3(4)$ & $\mathrm{C} 8-\mathrm{C} 9-\mathrm{C} 10-\mathrm{C} 11$ & $-3.1(4)$ \\
\hline $\mathrm{C} 1-\mathrm{C} 2-\mathrm{C} 3-\mathrm{S} 1$ & $175.60(19)$ & $\mathrm{C} 8-\mathrm{C} 9-\mathrm{C} 10-\mathrm{S} 2$ & $173.79(19)$ \\
\hline $\mathrm{C} 1-\mathrm{C} 2-\mathrm{C} 3-\mathrm{Ag} 2$ & $79.1(3)$ & $\mathrm{C} 8-\mathrm{C} 9-\mathrm{C} 10-\mathrm{Ag} 1^{\mathrm{iv}}$ & $78.3(3)$ \\
\hline $\mathrm{O} 3-\mathrm{S} 1-\mathrm{C} 3-\mathrm{C} 2$ & $98.3(2)$ & $\mathrm{O} 9-\mathrm{S} 2-\mathrm{C} 10-\mathrm{C} 9$ & $98.0(2)$ \\
\hline $\mathrm{O} 5-\mathrm{S} 1-\mathrm{C} 3-\mathrm{C} 2$ & $-24.1(2)$ & $\mathrm{O} 8-\mathrm{S} 2-\mathrm{C} 10-\mathrm{C} 9$ & $-24.5(2)$ \\
\hline $\mathrm{O} 4-\mathrm{S} 1-\mathrm{C} 3-\mathrm{C} 2$ & $-141.8(2)$ & $\mathrm{O} 10-\mathrm{S} 2-\mathrm{C} 10-\mathrm{C} 9$ & $-141.9(2)$ \\
\hline $\mathrm{Ag} 1-\mathrm{S} 1-\mathrm{C} 3-\mathrm{C} 2$ & $-82.2(2)$ & $\mathrm{Ag} 2-\mathrm{S} 2-\mathrm{C} 10-\mathrm{C} 9$ & $-82.8(2)$ \\
\hline $\mathrm{Ag} 2-\mathrm{S} 1-\mathrm{C} 3-\mathrm{C} 2$ & $-121.2(2)$ & $\mathrm{Ag} 1{ }^{\mathrm{ii}}-\mathrm{S} 2-\mathrm{C} 10-\mathrm{C} 9$ & $-178.88(17)$ \\
\hline $\mathrm{Ag} 2 \mathrm{v}-\mathrm{S} 1-\mathrm{C} 3-\mathrm{C} 2$ & $-179.76(17)$ & $\mathrm{Ag} 1^{\mathrm{iv}}-\mathrm{S} 2-\mathrm{C} 10-\mathrm{C} 9$ & $-121.3(2)$ \\
\hline $\mathrm{Ag} 2^{\mathrm{iii}}-\mathrm{S} 1-\mathrm{C} 3-\mathrm{C} 2$ & $-9.3(3)$ & $\mathrm{Ag} 1-\mathrm{S} 2-\mathrm{C} 10-\mathrm{C} 9$ & $-8.4(3)$ \\
\hline $\mathrm{Ag} 1-\mathrm{S} 1-\mathrm{C} 3-\mathrm{C} 2$ & $114.91(19)$ & $\mathrm{Ag} 2{ }^{\mathrm{ii}}-\mathrm{S} 2-\mathrm{C} 10-\mathrm{C} 9$ & $113.49(19)$ \\
\hline $\mathrm{O} 3-\mathrm{S} 1-\mathrm{C} 3-\mathrm{C} 4$ & $-83.7(2)$ & $\mathrm{O} 9-\mathrm{S} 2-\mathrm{C} 10-\mathrm{C} 11$ & $-85.1(2)$ \\
\hline $\mathrm{O} 5-\mathrm{S} 1-\mathrm{C} 3-\mathrm{C} 4$ & $153.9(2)$ & $\mathrm{O} 8-\mathrm{S} 2-\mathrm{C} 10-\mathrm{C} 11$ & $152.4(2)$ \\
\hline $\mathrm{O} 4-\mathrm{S} 1-\mathrm{C} 3-\mathrm{C} 4$ & $36.1(2)$ & $\mathrm{O} 10-\mathrm{S} 2-\mathrm{C} 10-\mathrm{C} 11$ & $35.0(2)$ \\
\hline $\mathrm{Ag} 1-\mathrm{S} 1-\mathrm{C} 3-\mathrm{C} 4$ & $95.7(2)$ & $\mathrm{Ag} 2-\mathrm{S} 2-\mathrm{C} 10-\mathrm{C} 11$ & $94.1(2)$ \\
\hline $\mathrm{Ag} 2-\mathrm{S} 1-\mathrm{C} 3-\mathrm{C} 4$ & $56.71(19)$ & $\mathrm{Ag} 11^{\mathrm{ii}}-\mathrm{S} 2-\mathrm{C} 10-\mathrm{C} 11$ & $-2.0(2)$ \\
\hline $\mathrm{Ag} 2 \mathrm{v}-\mathrm{S} 1-\mathrm{C} 3-\mathrm{C} 4$ & $-1.8(2)$ & $\mathrm{Ag} 1^{\mathrm{iv}}-\mathrm{S} 2-\mathrm{C} 10-\mathrm{C} 11$ & $55.63(18)$ \\
\hline $\mathrm{Ag}^{\mathrm{iii}}-\mathrm{S} 1-\mathrm{C} 3-\mathrm{C} 4$ & $168.65(14)$ & $\mathrm{Ag} 1-\mathrm{S} 2-\mathrm{C} 10-\mathrm{C} 11$ & $168.49(14)$ \\
\hline $\mathrm{Ag} 1-\mathrm{S} 1-\mathrm{C} 3-\mathrm{C} 4$ & $-67.1(2)$ & $\mathrm{Ag} 22^{\mathrm{ii}}-\mathrm{S} 2-\mathrm{C} 10-\mathrm{C} 11$ & $-69.6(2)$ \\
\hline $\mathrm{O} 3-\mathrm{S} 1-\mathrm{C} 3-\mathrm{Ag} 2$ & $-140.42(9)$ & $\mathrm{O} 9-\mathrm{S} 2-\mathrm{C} 10-\mathrm{Ag} 1^{\mathrm{iv}}$ & $-140.70(9)$ \\
\hline $\mathrm{O} 5-\mathrm{S} 1-\mathrm{C} 3-\mathrm{Ag} 2$ & $97.16(9)$ & $\mathrm{O} 8-\mathrm{S} 2-\mathrm{C} 10-\mathrm{Ag} 1^{\mathrm{iv}}$ & $96.78(9)$ \\
\hline $\mathrm{O} 4-\mathrm{S} 1-\mathrm{C} 3-\mathrm{Ag} 2$ & $-20.57(9)$ & $\mathrm{O} 10-\mathrm{S} 2-\mathrm{C} 10-\mathrm{Ag} 1^{\mathrm{iv}}$ & $-20.60(9)$ \\
\hline $\mathrm{Ag} 1-\mathrm{S} 1-\mathrm{C} 3-\mathrm{Ag} 2$ & $39.04(7)$ & $\mathrm{Ag} 2-\mathrm{S} 2-\mathrm{C} 10-\mathrm{Ag} 1^{\mathrm{iv}}$ & $38.49(7)$ \\
\hline $\mathrm{Ag} 2 \mathrm{v}-\mathrm{S} 1-\mathrm{C} 3-\mathrm{Ag} 2$ & $-58.52(6)$ & $\mathrm{Ag} 1^{\mathrm{ii}}-\mathrm{S} 2-\mathrm{C} 10-\mathrm{Ag} 1^{\mathrm{iv}}$ & $-57.59(6)$ \\
\hline $\mathrm{Ag} 2^{\mathrm{iii}}-\mathrm{S} 1-\mathrm{C} 3-\mathrm{Ag} 2$ & $111.94(8)$ & $\mathrm{Ag} 1-\mathrm{S} 2-\mathrm{C} 10-\mathrm{Ag} 1^{\mathrm{iv}}$ & $112.87(8)$ \\
\hline $\mathrm{Ag} 1{ }^{\mathrm{i}}-\mathrm{S} 1-\mathrm{C} 3-\mathrm{Ag} 2$ & $-123.85(7)$ & $\mathrm{Ag} 2^{\mathrm{ii}}-\mathrm{S} 2-\mathrm{C} 10-\mathrm{Ag} 1^{\mathrm{iv}}$ & $-125.22(7)$ \\
\hline $\mathrm{C} 2-\mathrm{C} 3-\mathrm{C} 4-\mathrm{C} 5$ & $0.7(4)$ & $\mathrm{C} 9-\mathrm{C} 10-\mathrm{C} 11-\mathrm{C} 12$ & $1.6(4)$ \\
\hline $\mathrm{S} 1-\mathrm{C} 3-\mathrm{C} 4-\mathrm{C} 5$ & $-177.23(19)$ & $\mathrm{S} 2-\mathrm{C} 10-\mathrm{C} 11-\mathrm{C} 12$ & $-175.29(19)$ \\
\hline $\mathrm{Ag} 2-\mathrm{C} 3-\mathrm{C} 4-\mathrm{C} 5$ & $-114.6(2)$ & $\mathrm{Ag} 1^{\mathrm{iv}}-\mathrm{C} 10-\mathrm{C} 11-\mathrm{C} 12$ & $-114.1(2)$ \\
\hline $\mathrm{C} 2-\mathrm{C} 3-\mathrm{C} 4-\mathrm{Ag} 2$ & $115.3(2)$ & $\mathrm{C} 9-\mathrm{C} 10-\mathrm{C} 11-\mathrm{Ag} 1^{\mathrm{iv}}$ & $115.7(2)$ \\
\hline $\mathrm{S} 1-\mathrm{C} 3-\mathrm{C} 4-\mathrm{Ag} 2$ & $-62.63(17)$ & $\mathrm{S} 2-\mathrm{C} 10-\mathrm{C} 11-\mathrm{Ag} 1^{\text {iv }}$ & $-61.23(17)$ \\
\hline $\mathrm{C} 3-\mathrm{C} 4-\mathrm{C} 5-\mathrm{C} 6$ & $1.5(4)$ & $\mathrm{C} 10-\mathrm{C} 11-\mathrm{C} 12-\mathrm{C} 13$ & $1.5(4)$ \\
\hline $\mathrm{Ag} 2-\mathrm{C} 4-\mathrm{C} 5-\mathrm{C} 6$ & $-99.0(2)$ & $\mathrm{Ag} 1^{\mathrm{iv}}-\mathrm{C} 11-\mathrm{C} 12-\mathrm{C} 13$ & $-99.3(2)$ \\
\hline
\end{tabular}




$\begin{array}{llll}\mathrm{C} 3-\mathrm{C} 4-\mathrm{C} 5-\mathrm{Ag} 2 & 100.5(3) & \mathrm{C} 10-\mathrm{C} 11-\mathrm{C} 12-\mathrm{Ag} 1^{\mathrm{iv}} & 100.8(3) \\ \mathrm{C} 4-\mathrm{C} 5-\mathrm{C} 6-\mathrm{C} 1 & -2.1(4) & \mathrm{C} 11-\mathrm{C} 12-\mathrm{C} 13-\mathrm{C} 8 & -3.0(4) \\ \mathrm{Ag} 2-\mathrm{C} 5-\mathrm{C} 6-\mathrm{C} 1 & -56.9(3) & \mathrm{Ag} 1 \mathrm{i}-\mathrm{C} 12-\mathrm{C} 13-\mathrm{C} 8 & -58.8(3) \\ \mathrm{C} 2-\mathrm{C} 1-\mathrm{C} 6-\mathrm{C} 5 & 0.5(4) & \mathrm{C} 9-\mathrm{C} 8-\mathrm{C} 13-\mathrm{C} 12 & 1.5(4) \\ \mathrm{C} 7-\mathrm{C} 1-\mathrm{C} 6-\mathrm{C} 5 & 179.0(2) & \mathrm{C} 14-\mathrm{C} 8-\mathrm{C} 13-\mathrm{C} 12 & -178.2(2) \\ \mathrm{C} 2-\mathrm{C} 1-\mathrm{C} 7-\mathrm{O} 1 & 163.7(3) & \mathrm{C} 13-\mathrm{C} 8-\mathrm{C} 14-\mathrm{O} 6 & 155.1(2) \\ \mathrm{C} 6-\mathrm{C} 1-\mathrm{C} 7-\mathrm{O} 1 & -14.8(4) & \mathrm{C} 9-\mathrm{C} 8-\mathrm{C} 14-\mathrm{O} 6 & -24.6(4) \\ \mathrm{C} 2-\mathrm{C} 1-\mathrm{C} 7-\mathrm{O} 2 & -16.4(3) & \mathrm{C} 13-\mathrm{C} 8-\mathrm{C} 14-\mathrm{O} 7 & -25.5(3) \\ \mathrm{C} 6-\mathrm{C} 1-\mathrm{C} 7-\mathrm{O} 2 & 165.0(2) & \mathrm{C} 9-\mathrm{C} 8-\mathrm{C} 14-\mathrm{O} 7 & 154.8(2)\end{array}$

Symmetry codes: (i) $-x+3,-y+1,-z+1$; (ii) $-x+2,-y,-z+1$; (iii) $x+1, y, z$; (iv) $x-1, y, z$; (v) $-x+2,-y+1,-z+1$.

Hydrogen-bond geometry $\left(\AA,{ }^{\circ}\right)$

\begin{tabular}{|c|c|c|c|c|}
\hline$D-\mathrm{H} \cdots A$ & $D-\mathrm{H}$ & $\mathrm{H} \cdots A$ & $D \cdots A$ & $D-\mathrm{H}^{\cdots} \cdot A$ \\
\hline $\mathrm{O} 2-\mathrm{H} 2 \cdots \mathrm{O} 1^{\mathrm{vi}}$ & 0.84 & 1.81 & $2.631(3)$ & 164 \\
\hline $\mathrm{O} 7-\mathrm{H} 7 \cdots \mathrm{O}^{\text {vii }}$ & 0.84 & 1.81 & $2.651(3)$ & 176 \\
\hline $\mathrm{C} 4-\mathrm{H} 4 \cdots \mathrm{O}^{v}$ & 0.95 & 2.43 & $3.214(3)$ & 140 \\
\hline $\mathrm{C} 11-\mathrm{H} 11 \cdots \mathrm{O} 5^{\mathrm{ii}}$ & 0.95 & 2.48 & $3.269(3)$ & 141 \\
\hline
\end{tabular}

Symmetry codes: (ii) $-x+2,-y,-z+1$; (v) $-x+2,-y+1,-z+1$; (vi) $-x+2,-y,-z$; (vii) $-x+2,-y+1,-z+2$. 